\title{
Analyzing an Electronic Limit Order Book
}

by David Kane, Andrew Liu, and Khanh Nguyen

\begin{abstract}
The orderbook package provides facilities for exploring and visualizing the data associated with an order book: the electronic collection of the outstanding limit orders for a financial instrument. This article provides an overview of the orderbook package and examples of its use.
\end{abstract}

\section{Introduction}

The orderbook package provides facilities for exploring and visualizing the data associated with an order book: the electronic collection of the outstanding limit orders for a financial instrument, e.g. a stock. A limit order is an order to buy or sell a given quantity of stock at a specified limit price or better. The size is the number of shares to be bought or sold. An order remains in the order book until fully executed, i.e. until its size is zero as a result of trades. Partial executions occur as a result of trades for less than the entire size of the order.

Consider a simple order book containing five limit orders: sell 150 shares of IBM at $\$ 11.11$, sell 150 shares of IBM at $\$ 11.08$, buy 100 shares of IBM at $\$ 11.05$, buy 200 shares of IBM at $\$ 11.05$, and buy 200 shares of IBM at $\$ 11.01$.

$\begin{array}{rlr} & \text { Price } & \text { Ask Size } \\ & \$ 11.11 & 150 \\ 300 & \$ 11.08 & 100 \\ 200 & \$ 11.05 & \\ & \$ 11.01 & \\ \text { Bid Size } & \text { Price } & \end{array}$

Orders on the bid (ask) side represent orders to buy (sell). The price levels are \$11.11, \$11.08, \$11.05, and $\$ 11.01$. The best bid at $\$ 11.05$ (highest bid price) and the best ask at $\$ 11.08$ (lowest ask price) make up the inside market. The spread (\$0.03) is the difference between the best bid and best ask. The midpoint (\$11.065) is the average of the best bid and best ask.

There are four types of messages that traders can submit to an order book: add, cancel, cancel/replace, and market order. A trader can add a limit order in to the order book. She can also cancel an order and remove it from the order book. If a trader wants to reduce the size of her order, she can issue a cancel/replace, which cancels the order, then immediately replaces it with another order at the same price, but with a lower size. Every limit order is assigned a unique ID so that cancel and cancel/replace orders can identify the corresponding limit order. A market order is an order to immediately buy or sell a quantity of stock at the best available prices. A trade occurs when a market order "hits" a limit order on the other side of the inside market.

All orders have timestamps indicating the time at which they were accepted into the order book. The timestamp determines the time priority of an order. Earlier orders are executed before later orders. For example, suppose that the order to buy 100 shares at $\$ 11.05$ was submitted before the order to buy 200 shares at $\$ 11.05$. Now suppose a market order selling 200 shares is submitted to the order book. The limit order for 100 shares will be executed because it is at the front of the queue at the best bid. Then, 100 shares of the order with 200 total shares will be executed, since it was second in the queue. 100 shares of the 200 share order remain in the order book at $\$ 11.05$.

A market order for more shares than the size at the inside market will execute at worse price levels until it is complete. For example, if a market order to buy 200 shares is submitted to the order book, the order at $\$ 11.08$ will be fully executed. Since there are no more shares available at that price level, 100 shares at the $\$ 11.11$ price level will be transacted to complete the market order. An order to sell 50 shares at $\$ 11.11$ will remain in the order book. Executing these two market orders (a sell of 200 shares and a buy of 200 shares) on our hypothetical order book results in a new state for the order book.

$\begin{array}{lll} & \text { Price } & \text { Ask Size } \\ 100 & \$ 11.11 & 50 \\ 200 & \$ 11.05 & \\ \text { Size } & \$ 11.01 & \\ & \text { Price } & \end{array}$

Note that cancel/replace orders can lower the size of an order, but not increase it. Cancel/replace orders maintain the time priority of the original order, so if size increases were allowed, traders with orders at the highest time priority for a price level could perpetually increase the size of their order, preventing others from being able to transact stock using limit orders at that price level. See Johnson (2010) for more details on the order book.

\section{Example}

NVIDIA is a graphics processing unit and chipset developer with ticker symbol NVDA. Consider the order book for NVDA at a leading electronic exchange 
on June 8,2010 . We create the orderbook object by specifying the location of our data file.

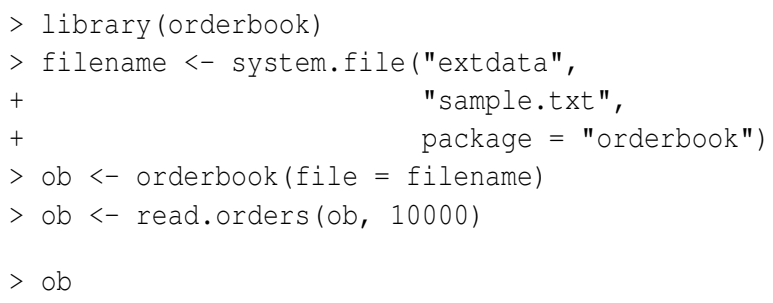

We read in the first 10,000 messages then show the object. The current time is 9:35:02 AM. This is the time of the last message read. The message index indicates which row in the data file the object has read through. The display also shows that there are 631 bids and 1,856 asks outstanding, for a total of 2,487 orders. This indicates that many earlier orders have been removed through either cancels or trades.

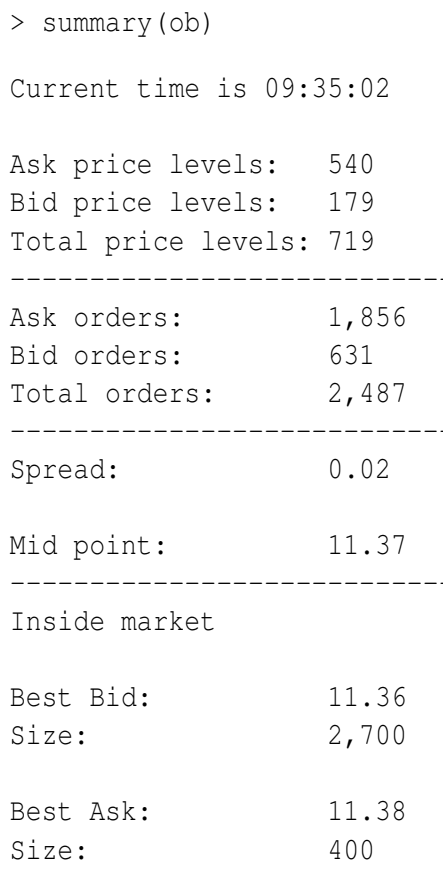

Using summary the total order information from show is repeated. Additionally, we see that there are 540 ask and 179 bid price levels, for a total of 719 . This indicates that many orders have been submitted at the same price level. The spread is $\$ 0.02$, and the midpoint is $\$ 11.37$. The inside market is composed of 2,700 shares offered at the best bid of $\$ 11.36$ and 400 shares offered at the best ask of $\$ 11.38$.

\begin{tabular}{|c|c|c|}
\hline \multicolumn{3}{|c|}{ Current time is 09:35:02 } \\
\hline & Price & Ask Size \\
\hline & 11.42 & 900 \\
\hline & 11.41 & 1,400 \\
\hline & 11.40 & 1,205 \\
\hline & 11.39 & 1,600 \\
\hline & 11.38 & 400 \\
\hline 2,700 & 11.36 & \\
\hline 1,100 & 11.35 & \\
\hline 1,100 & 11.34 & \\
\hline 1,600 & 11.33 & \\
\hline 700 & 11.32 & \\
\hline
\end{tabular}

Bid Size Price

display shows the inside market, along with the four next best bid and ask price levels and the size at each price level.

$>$ plot (ob)

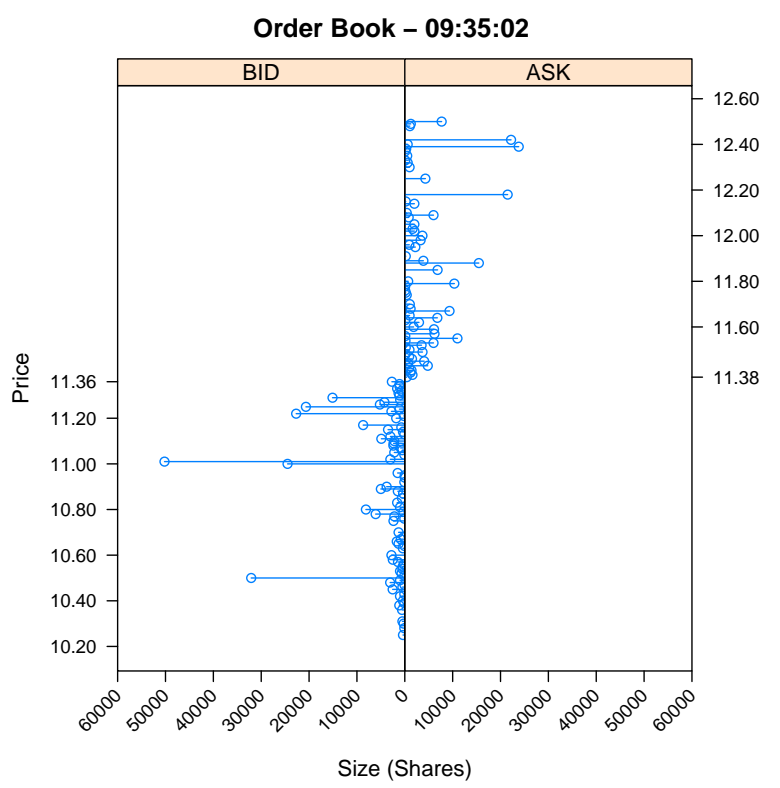

plot is a graphical representation of display. Price levels are on the $y$-axis, and size is on the $x$-axis. The maximum and minimum price levels displayed by default are $10 \%$ above and below the midpoint. Note the large number of shares at $\$ 11.01$. It is helpful to know the number of orders which make up the large size at that price level. Using the " [" method we can view the order information at particular price levels. 


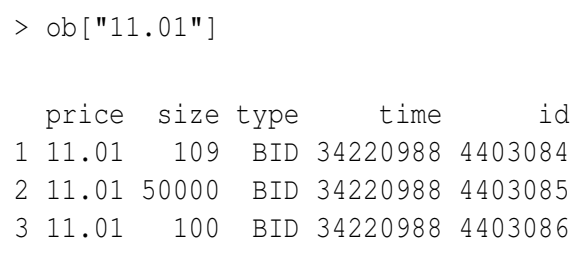

There is an order for 50,000 shares at the $\$ 11.01$ price level that accounts for almost all of the size. We can view a plot of the number of orders rather than the number of shares at each price level by specifying type $={ }^{\prime} \circ$ ' when using plot. In the previous plot the maximum and minimum price levels were $10 \%$ off from the midpoint, but for this plot we specify a range of only $3.3 \%$.

Note the large number of orders at $\$ 11.00$. The " [ " method returns a data. frame, so we can use nrow to return the number of orders at $\$ 11.00$.

$>\operatorname{nrow}(0 \mathrm{~b}[11.00 "])$

[1] 56

There are 56 orders at that price level, which confirms what we see in the plot.

$>$ plot $\left(\mathrm{ob}\right.$, bounds $=0.033$, type $=\mathrm{\prime}^{\prime}$ ')

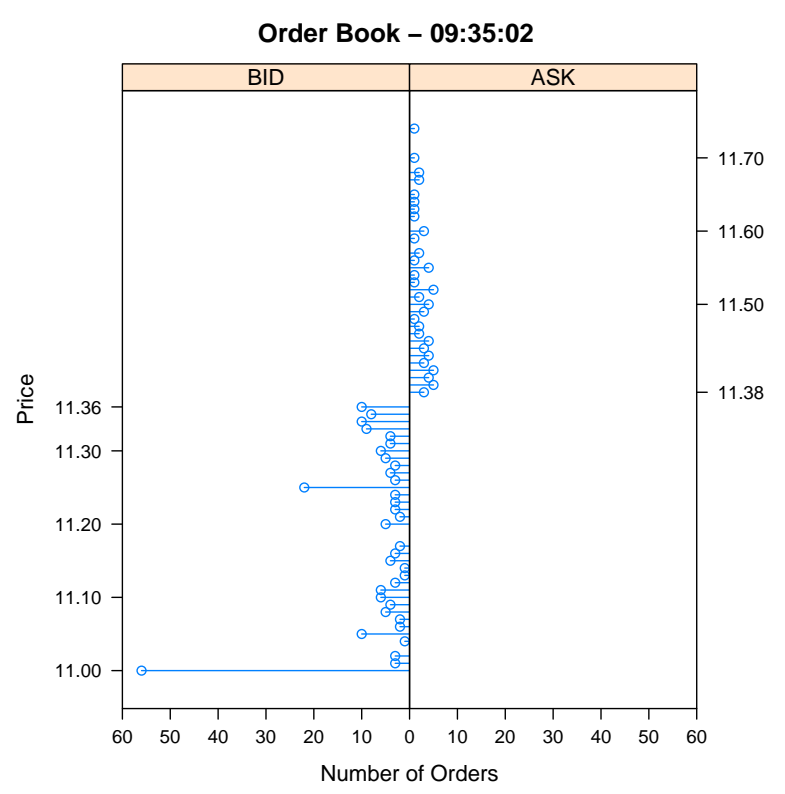

The type argument on plot allows for an "sd" option which shows supply and demand curves for the order book. The demand (supply) curve is downsloping (upsloping). This is because more people want to buy (sell) a stock when the price decreases (increases). The ask (bid) prices are normalized by the absolute value of the difference between the highest (lowest) plotted ask (bid) price level and the the midpoint. Following Cao et al. (2009), the sizes are normalized by the sum of the sizes across all plotted price levels for each side.

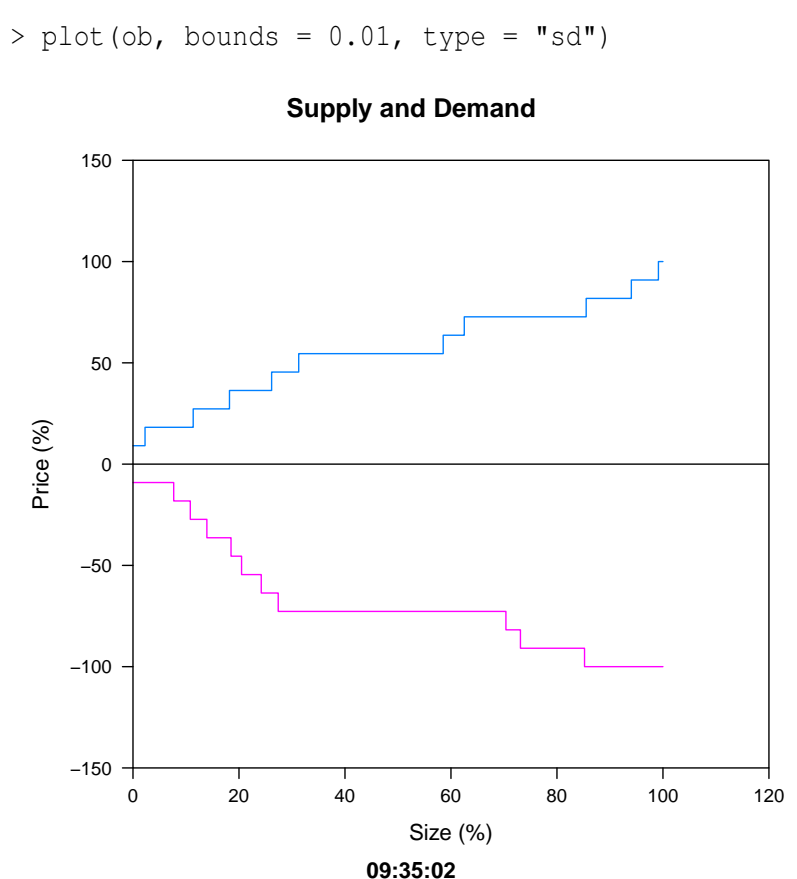

orderbook has methods for creating new orderbook objects at specified clock times of interest. read.time returns an orderbook object that has read all messages before the specified time. For example, this returns the orderbook object at 9:30:00.

$>$ ob <- read.time (ob, "9:30:00")

read.orders is used to move forwards or backwards in the order book by a specified number of messages. In this case, an orderbook object at 50 messages before the current message is returned.

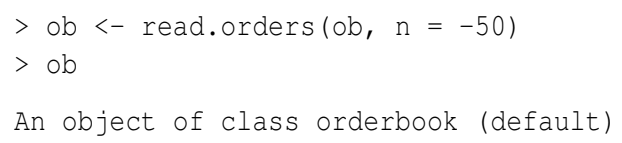

\section{Data}

Data files should contain all messages for one stock on a single trading day. Most brokers and exchanges have their own format for transmitting raw message data to customers, so it would be unfeasible for us to write scripts to automatically process all data formats. Consequently, raw data for an orderbook object must be in the following form:

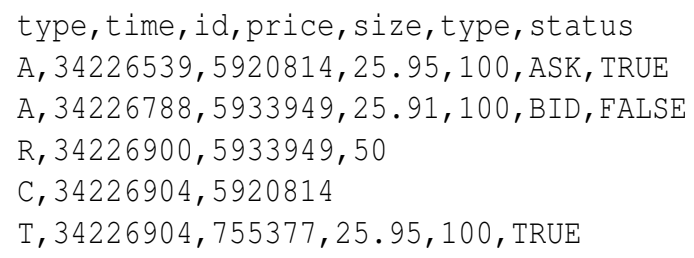


where A, R, T, and C mean Add, Replace, Trade, and Cancel, respectively. The second column is the timestamp of the message in milliseconds after midnight, and the third column is the order ID. For a Replace the next column is the new size, while for Add and Trade a column for price comes before the size column. Add messages also have the type of order (BID/ASK) in the sixth column. The optional seventh (sixth) column is TRUE if the order (trade) belongs to the user, and FALSE otherwise. This allows the user to create plots that show the time priority of his own orders. If the column is omitted, the first line of the data file should be type, time, id, price, size, type and not include status.

In this example a user order to sell 100 shares at $\$ 25.95$ is added to the order book, followed by an order to buy 100 shares at $\$ 25.91$. The size of the order at $\$ 25.91$ is then replaced to 50 shares. Finally, the order at \$25.95 is cancelled, and a trade for 100 shares at $\$ 25.95$ occurs.

\section{Analyzing trades}

A user can create plots that show the time priority of his own orders if a status column is present in the data file.

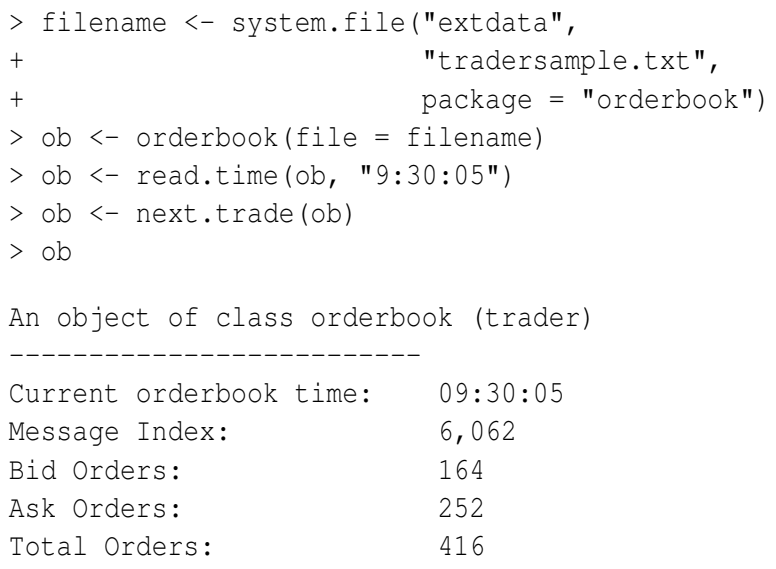

Note that this orderbook object is of type trader. The next.trade function sets the state of the order book to when the trade after the current time occurs. There is also a previous.trade function with the same functionality moving backwards

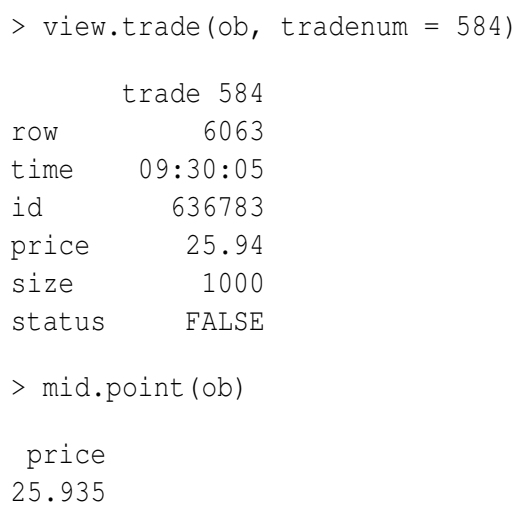

Since the trade price is higher than the midpoint price, we know that the trade occurred as a result of an ask order getting hit. Note that trade data is stored into the order book only after it has read through the corresponding trade message.

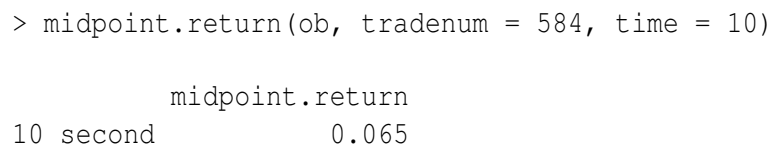

The midpoint return is the difference in cents between the execution price and the midpoint price after a specified period of time. For example, the above calculates the ten second midpoint return for the first trade. Since it was a sell order, the midpoint return will be positive if the stock price decreases, and negative if the stock price increases.
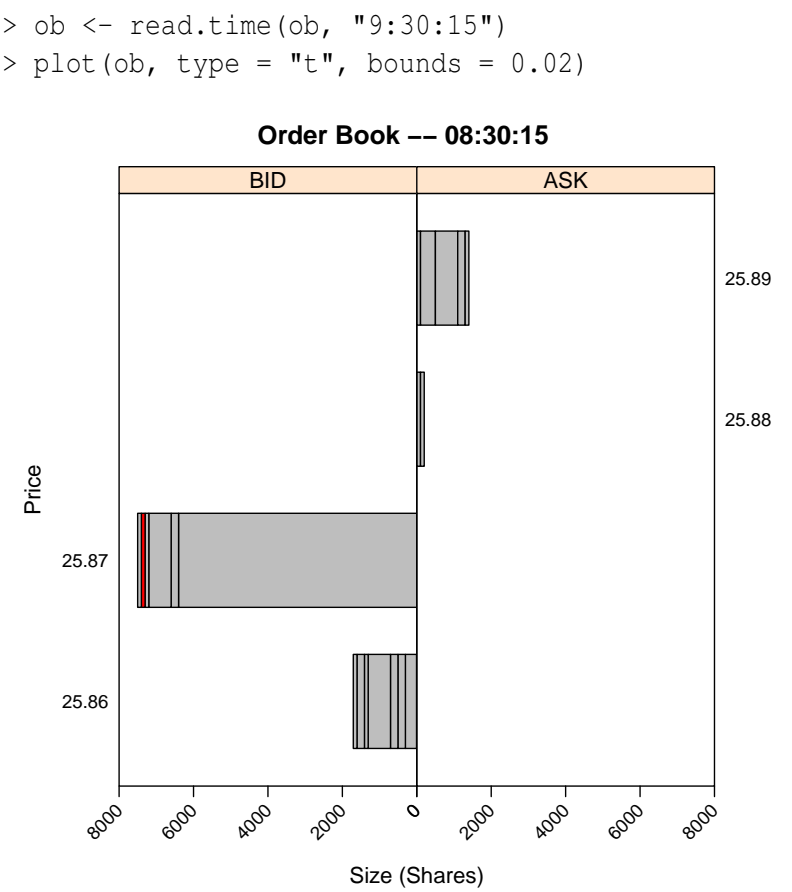

This plot shows two pennies above and below the best bid and best ask. We see that the midpoint has dropped to 25.875, confirming the midpoint return above. This graph shows two pennies above and below the best bid and ask. Orders at these price levels are shown in time priority, with the earliest submitted order being closest to the middle y-axis. Note the red order-this is an order marked TRUE by the user, indicating that it belonged to him.

\section{Simulation}

Simulating and modelling the intraday decisions of traders is a topic of active research in behavioral finance and economics. orderbook supports adding, replacing, and cancelling orders. Add orders require the price, size, and type (ASK/BID) of the limit order. Time and ID are optional, and will default to the 
maximum time +1 and the maximum ID +1 . Replace messages require the new size and ID. Cancel orders only require ID. In addition, market orders can be issued to the order book. Market orders require size and side (BUY/SELL).

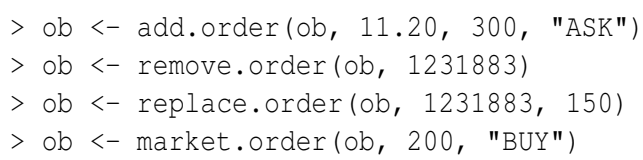

Using these tools, the user can write functions to simulate the an order book. In the following example, we consulted Gilles (2006). We simulate 1,000 messages. The messages are chosen based on the following probabilities: $50 \%$ for a cancel message, $20 \%$ for a market order, and $30 \%$ for a limit order. In the event of a cancel message the order cancelled is randomly chosen. Market order have a 50-50 chance for a buy or sell order. The size of the market order always corresponds to the size of the individual order at the best ask or bid with the highest time priority. Limit orders have a 50-50 chance to be an ask or bid. There is a $35 \%$ chance for the price of a limit order to be within the spread. If the price is outside of the spread, a price is chosen using a power law distribution. Finally, the size follows a log-normal distribution. A plot of this example simulation is shown below.
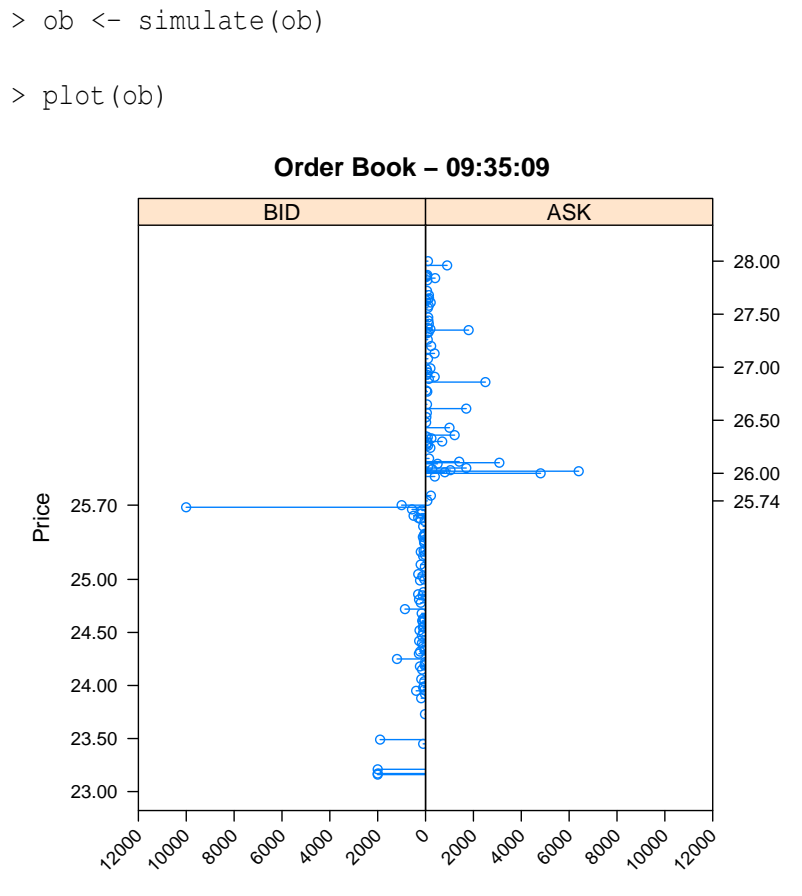

Size (Shares)
Gilles (2006) used simulations to test the impact of liquidity on price level stabilization. He concluded that most price changes are the result of uninformed traders (who supply liquidity), rather than informed traders (who demand liquidity).

\section{Conclusion}

The orderbook package is part of a collection of packages (see Campbell et al. (2007) and Kane and Enos (2006)) for working with financial market data. $R$ provides all the necessary tools for managing institutional sized portfolios.

\section{David Kane}

Kane Capital Management

Cambridge, $M A$

USA

dave@kanecap.com

Andrew Liu

Williams College

Williamstown, $M A$

USA

Andrew.T.Liu@williams.edu

Khanh Nguyen

University Of Massachusetts at Boston

Boston, $M A$

USA

knguyendes. umb. edu

\section{Bibliography}

K. Campbell, J. Enos, D. Gerlanc, and D. Kane. Backtests. R News, 7(1):36-41, April 2007.

C. Cao, O. Hansch, and X. Wang. The information content of an open limit-order book. The Journal of Futures Markets, 29(1):16-41, 2009.

D. Gilles. Asynchronous Simulations of a Limit Order Book. PhD thesis, University of Manchester, 2006.

B. Johnson. Algorithmic Trading \& DMA: An introduction to direct access trading strategies. 4Myeloma Press, London, 2010.

D. Kane and J. Enos. Analysing equity portfolios in R. R News, 6(2):13-19, May 2006. 